\title{
Extended Dwell Time Improves Results of Fibrinolytic Therapy for Complex Pleural Effusions
}

\author{
Sanja H. Patino ${ }^{1}$, Francisco Tarrazzi ${ }^{2}$, Catherine Tami ${ }^{2}$, Alyssa Bellini ${ }^{3}$, Mark Block ${ }^{2}$ \\ 1. Department of Internal Medicine, Florida Atlantic University Charles E. Schmidt College of Medicine, Boca Raton, \\ USA 2. Division of Thoracic Surgery, Memorial Healthcare, Hollywood, USA 3. Department of General Surgery, \\ University of California Davis School of Medicine, Sacramento, USA
}

Corresponding author: Sanja H. Patino, shotic2013@health.fau.edu

\section{Abstract}

\section{Introduction}

Published trials of intrapleural therapy for complex pleural effusions rely on fibrinolytics and deoxyribonuclease (DNase) with dwell times of less than six hours and frequent dosing. We reviewed our experience with fibrinolytics alone but with a longer dwell time (12 hours).

\section{Methods}

Tissue plasminogen activator (tPA, 1-6 mg per dose) was given through pigtail catheters placed using image guidance. Planned treatment was for a dwell time of 12 hours with repeat dosing daily for three days or until drainage was less than $100 \mathrm{cc}$ or grossly bloody. Chest $\mathrm{x}$-ray and/or computed tomography (CT) were used to determine completeness of pleural drainage.

\section{Results}

Forty-six patients presenting with 47 complex pleural effusions were given 131 doses of tPA. Doses of 4, 5, and $6 \mathrm{mg}$ were most common ( $\mathrm{n}=17,70$, and 33 , respectively). Dwell time ranged from five to 14 hours with 12 hours being most common ( $\mathrm{n}=115$ ). Additional chest tubes were placed in 18 effusions. Ten effusions (21\%) required decortication: seven for trapped lung and three for incomplete drainage. Drainage was considered complete in $33 / 40$ (82.5\%) effusions without trapped lung. Median chest tube duration was seven days (range three to 28 days). tPA therapy was discontinued in two patients for bleeding, but neither experienced hemodynamic instability.

\section{Conclusions}

tPA with a 12-hour dwell time is effective and safe for management of complex pleural effusions, although chest tube duration was prolonged. tPA alone is less expensive and easier than when combined with DNase,

Received 07/22/2020 Review began $07 / 27 / 2020$ Review ended 07/27/2020 Published 08/11/2020

\section{(c) Copyright 2020}

Patino et al. This is an open access article distributed under the terms of the Creative Commons Attribution License CC-BY 4.0., which permits unrestricted use, distribution, and reproduction in any medium, provided the original author and source are credited.
Categories: Cardiac/Thoracic/Vascular Surgery, Internal Medicine, Pulmonology

Keywords: pleural effusion, tissue plasminogen activator, intrapleural therapy, tpa, chest tube, complex pleural effusion

\section{Introduction}

Intrapleural fibrinolytic therapy can be effective for management of complex pleural effusions that do not respond to thoracentesis [1]. These effusions are typically exudative, with patients presenting in the second, or fibrinopurulent, phase of development [2]. This phase begins about two weeks after the initiating event and lasts for about four to six weeks. Fibrin deposition creates loculated, complex fluid collections that are no longer amenable to simple drainage, ultimately necessitating decortication $[1,2]$. However, there has been longstanding interest in fibrinolytic therapy instead of surgery when the lung is not "trapped" from organizing fibrosis. Initial experience with intrapleural urokinase and streptokinase supported this concept [3-8]. Tissue plasminogen activator (tPA) is a naturally occurring fibrinolytic, now available as a recombinant protein, and is approved for management of acute thrombotic events such as stroke and coronary artery thrombosis. It is also effective as an intrapleural fibrinolytic agent and recent protocols suggest frequent dosing in combination with DNase for best results [9-11].

Our service frequently manages patients with complex pleural effusions and started using tPA in 2012. DNase was expensive and not readily available, and therefore was not used. Through a serendipitous observation after a chest tube was left clamped overnight, it was discovered that the prolonged dwell time yielded significant drainage. As a result, we implemented a strategy of daily tPA alone with a dwell time of 12 hours. The anecdotal experience seemed encouraging, with the majority of patients achieving effective 
drainage. Therefore, we undertook a retrospective review to determine if tPA alone with a prolonged dwell time is sufficiently effective to warrant prospective evaluation.

\section{Materials And Methods}

Institutional Review Board approval was obtained for this retrospective review. Charts were reviewed for patients with complex pleural effusions that were treated with tPA between November 2012 and April 2016. Demographics and clinical data were collected, and imaging results recorded.

\section{Chest tube placement and tPA therapy}

Pigtail catheters ( $8-14 \mathrm{Fr}$ ) were placed into the target effusions using image guidance (CT or ultrasound) and allowed to drain spontaneously. tPA (1-6 mg) in $50 \mathrm{ml}$ of normal saline was given at bedside through the chest tube and the tube was clamped with nursing orders to unclamp after a dwell time of 12 hours. The following day drainage was assessed and a determination was made about whether to repeat treatment. In general, additional doses were given if the drainage was more than $100 \mathrm{ml}$ and not grossly bloody. Target therapy was for three doses over three days. Chest x-ray and chest CT were used to evaluate completeness of drainage. If a residual loculated effusion separate from the drainage catheter was identified, an additional drain was placed and tPA therapy continued. These decisions were made by the individual clinicians and were not protocol-driven.

\section{Outcome assessment}

Outcomes were assigned to one of four groups based on imaging: 1) complete drainage, defined as trace to no residual pleural fluid, 2) trapped lung, defined as minimal residual pleural fluid but incomplete expansion of the lung with a pneumothorax replacing the pleural effusion, 3) incomplete drainage without surgery, defined as moderate residual pleural effusion but a clinical decision not to proceed with decortication, and 4) incomplete drainage with surgery, defined as a moderate residual pleural effusion with the clinical decision to proceed with decortication.

\section{Results}

\section{Patient characteristics}

Review of medical records identified 46 patients with 47 effusions (one patient had bilateral effusions) treated with at least one dose of tPA (Table 1). Most patients were male, and most effusions were right-sided. Twenty-one effusions were parapneumonic (sterile) and 16 were empyemas with positive pleural fluid cultures (appendix). The most common presenting symptom was dyspnea and many patients had a combination of symptoms (appendix). Symptoms were present on average seven days before initiation of fibrinolytic therapy (range 1 to 30 ). 


\section{Cureus}

Age (years)

Mean \pm SD

$60 \pm 13.9$

Range

$24-92$

Gender, n

Female

$13(28 \%)$

Male

Laterality, $\mathrm{n}$

Right

$27(59 \%)$

Left

$19(41 \%)$

Etiology, $\mathrm{n}$

Parapneumonic

$21(45 \%)$

Empyema

$16(34 \%)$

Reactive, intra-abdominal process

2

Esophageal perforation

Atelectasis

Pleuritis

1

Malignancy

1

Unknown

2

\section{TABLE 1: Patient Demographics}

\section{Thrombolytic therapy}

Chest tube sizes for initial drainage ranged from 8 to $14 \mathrm{Fr}$, with the large majority of catheters 8 or $10 \mathrm{Fr}$ (Table 2). A second chest tube was placed in 17 cases, and a third chest tube was placed in one. Fibrinolytic therapy with tPA was delivered 131 times (average 2.7 per patient) in doses ranging from 1 to $6 \mathrm{mg}$ (Figure 1). Sixty-six percent of effusions received two or three doses and $92 \%$ of the doses were 4 to $6 \mathrm{mg}$. For the vast majority of doses, the target dwell time of 12 hours was achieved (Table 3). However, on nine occasions, symptoms such as pressure and dyspnea prompted nursing to unclamp the tubes earlier.

\begin{tabular}{|c|c|c|}
\hline Chest Tube Size (French) & First Tube (N) & Additional Tube (N) \\
\hline 8 & 30 & 11 \\
\hline 10 & 16 & 5 \\
\hline 12 & 0 & 1 \\
\hline 14 & 1 & 0 \\
\hline 20 & 0 & 1 \\
\hline
\end{tabular}

TABLE 2: Chest Tube Size 


\section{Cureus}
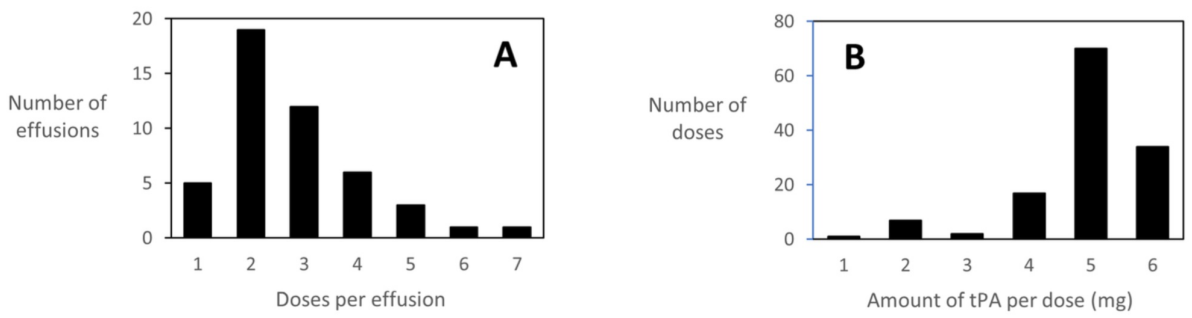

FIGURE 1: Summary of tPA Dosing for Management of 47 Complex Pleural Effusions

A) Number of effusions treated with each number of doses of tPA. Note that five effusions were treated with only one dose of tPA. B) Number of times each amount of tPA was used per dose.

tPA: tissue plasminogen activator

Dwell Time (hours)

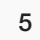

6

7

10

11

12

14

Not documented
Number of Doses

1

4

1

115

1

6

TABLE 3: Dwell Time

\section{Chest tube drainage}

Drainage following tPA seemed dose-independent (Figure 2). Duration of therapy was most often three days and $66 \%$ of effusions had therapy completed within five days (range 1-15 days, Figure 3). Chest tube drainage was complete by eight days in $68 \%$ of cases, and by 11 days in $89 \%$ of cases. In one case, chest tube drainage continued for 28 days. 


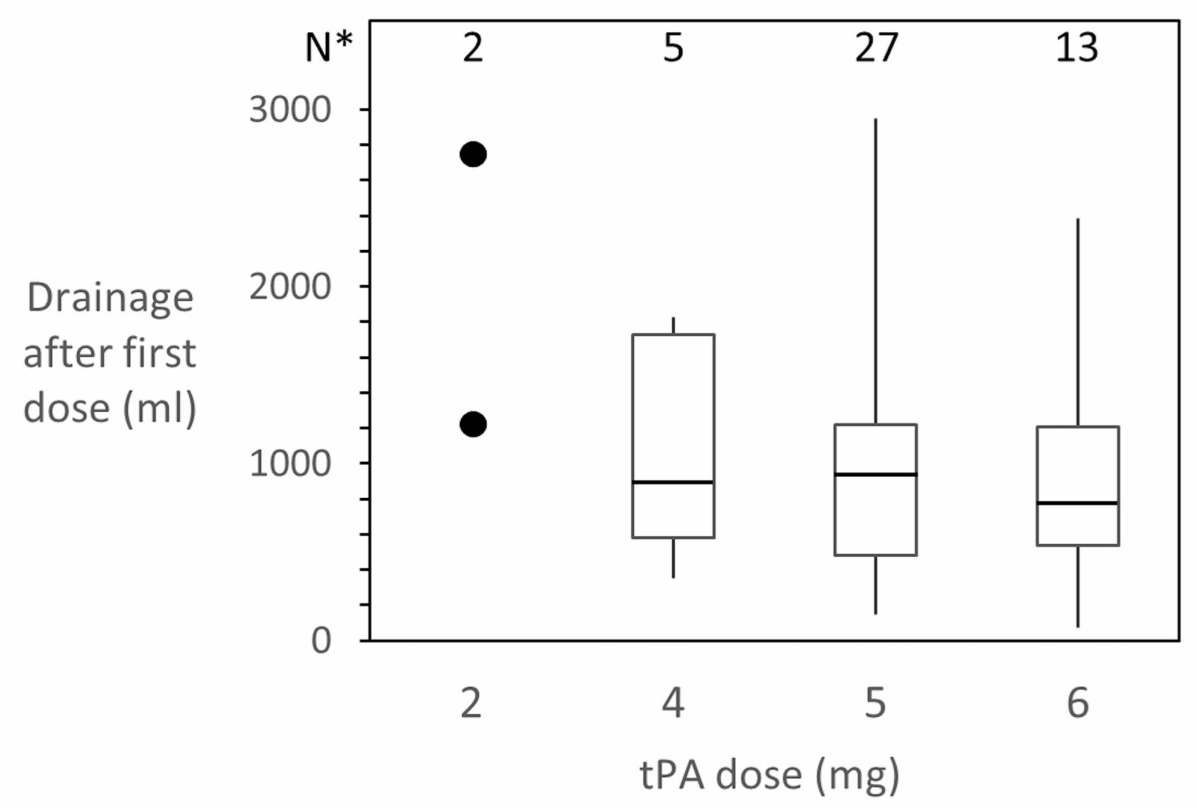

FIGURE 2: Volume of Pleural Fluid Drained After the First Dose of tPA

Range, median and interquartile range for drainage volume measured from when the chest tube was unclamped to when the second dose was given. For patients given only one dose, this is the first 24 hours after the tube was unclamped. Two patients were given $2 \mathrm{mg}$, with drainage recorded as $460 \mathrm{ml}$ and $3500 \mathrm{ml}$. * Number of patients in each group. tPA: tissue plasminogen activator

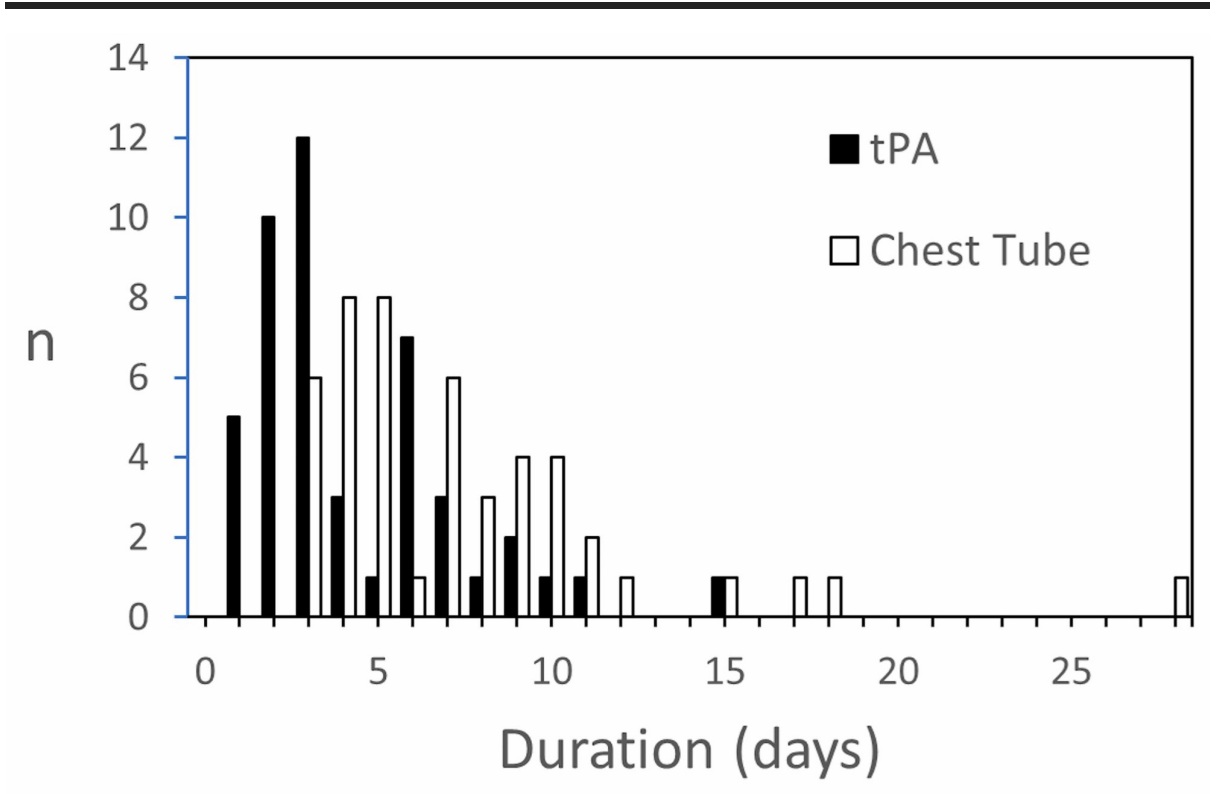

\section{FIGURE 3: Duration of Therapy}

Filled bars are for time from the first dose of tPA to the last. Open bars are for time from the first dose of tPA to when the last chest tube was removed. $n=$ number of effusions. tPA: tissue plasminogen activator

\section{Outcome}

Post-treatment chest CT scans were available for 41 effusions (87\%). For the remaining six, assessment of treatment success was made based on chest $\mathrm{x}$-rays. Most effusions (70\%) were assessed to have been completely drained (Figure 4). Of the remaining 30\%, half had a trapped lung and the other half had incomplete drainage. Only three cases, half of the effusions with incomplete drainage, underwent decortication. Complete drainage was associated with the shortest symptom duration while trapped lung was associated with the longest (Table 4). 


\section{Cureus}

Incomplete: Surgery

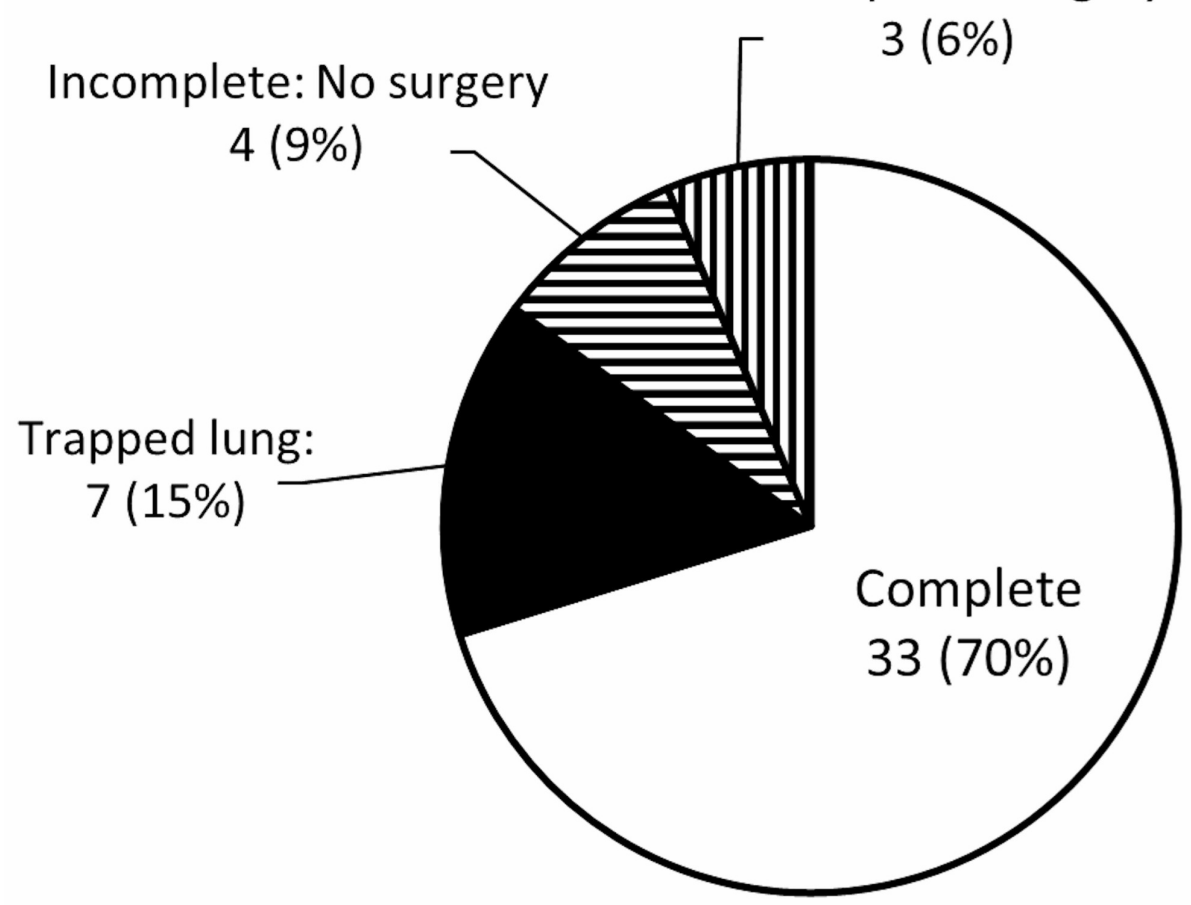

FIGURE 4: Outcomes of tPA Therapy for 47 Pleural Effusions

tPA: tissue plasminogen activator

\begin{tabular}{|l|l|l|}
\hline Outcome & N & Symptom Duration, days Mean (Range) \\
\hline Complete drainage & 33 & $6(1-21)$ \\
\hline Trapped lung & 7 & $12(2-30)$ \\
Incomplete drainage, no surgery & 4 & $5(2-7)$ \\
Incomplete drainage, surgery & 3 & $12(7-21)$ \\
\hline
\end{tabular}

\section{TABLE 4: Symptom Duration by Outcome}

\section{Discussion}

The goal of intervention for patients presenting with an exudative pleural effusion is to achieve effective drainage with complete lung expansion and relief of symptoms. While decortication can accomplish this goal quickly, an attractive alternative is intrapleural fibrinolytic therapy. If successful, it avoids the morbidity and cost associated with surgery. If unsuccessful, it delays necessary surgery and prolongs hospitalization. We reviewed our experience with a simple regimen of tPA alone with a prolonged dwell time and found that it was successful in the majority of cases, although in some cases treatment duration was prolonged.

Intrapleural fibrinolytic therapy was first described by Tillet and associates in 1951, who reported using streptokinase and streptococcal deoxyribonuclease (DNase) to facilitate pleural drainage in patients with empyema [3]. Streptokinase, and fibrinolytics in general, dissolve fibrinous clots and membranes to prevent fluid sequestration while DNase destroys extracellular DNA, which is thought to help prevent biofilm formation and reduce fluid viscosity. Subsequently, several small controlled studies reported the efficacy of streptokinase and urokinase for improving pleural drainage [4-8]. The limitations of these small studies led to the first Multicenter Intrapleural Sepsis Trial (MIST-1), a large randomized placebo-controlled trial of fibrinolytic therapy published in 2005. MIST-1 found that there was no benefit with streptokinase in radiographic outcomes, need for surgery, survival, or length of hospital stay, but that there were more adverse events [12]. These findings were later supported by a meta-analysis [13]. Although the MIST-1 trial 
found no benefit, the proportion of enrolled patients who had loculated pleural effusions was low. Therefore, because of otherwise strong clinical and laboratory evidence, interest in intrapleural fibrinolytic therapy continued and research efforts shifted toward the use of recombinant tPA, a direct-acting fibrinolytic agent [14].

There have been several studies supporting the use of intrapleural tPA in adults, however there is wide variation in the number of enrolled patients, number of daily doses, the dose used, and the dwell time of tPA in the pleural space [15-18]. In 2011, the second Multicenter Intrapleural Sepsis Trial (MIST-2) included a comparison of tPA with DNase [9]. MIST-2 found that a twice-daily combination of $10 \mathrm{mg}$ of tPA and $5 \mathrm{mg}$ of DNase with a dwell time of one hour produced a greater decrease in pleural opacity by day seven, decreased surgery referral, and reduced hospital stay relative to double placebo, DNase, or tPA alone [9]. In 2014, Piccolo and colleagues reported improved clearance of pleural opacities in a retrospective series of 107 patients from eight institutions treated with the same combined regimen [10]. In 2016, Mehta and colleagues reported good results in 51 of 55 patients treated with three days of once-daily intrapleural tPA/DNase (10 and $5 \mathrm{mg}$ respectively) and one-hour dwell time [11]. As a result of these studies, current practice for intrapleural therapy for complex pleural effusions is frequent dosing with a combination of tPA and DNase and short dwell times. Because these patients are often distributed across the hospital on different nursing units with varying degrees of expertise with chest tube care, this approach can require significant time commitment from providers, limiting its feasibility. In our experience, based on a serendipitous observation, once-daily therapy with tPA alone and a prolonged dwell time of 12 hours achieved effective drainage in the large majority of cases. This strategy is logistically simpler and less workintensive, and therefore much more feasible.

For tPA to be a viable alternative to standard surgical therapy, it should achieve similar results with less morbidity and cost. Thoracoscopy is usually associated with minimal morbidity and short hospital stays but is inadequate in the setting of trapped lung, which generally requires thoracotomy for complete decortication. Our results demonstrate that a short course of tPA with a prolonged dwell time achieved the goals of therapy in the large majority (82.5\%) of patients who did not have trapped lung, suggesting that it is a viable alternative to thoracoscopy. We also found that tPA was beneficial in those patients with trapped lung because it enabled rapid diagnosis. As expected, symptom duration before presentation was longest in patients with trapped lung, but this factor alone is insufficient for diagnosis. Rapid diagnosis facilitates prompt decision making between proceeding with the more invasive thoracotomy or delaying surgery to proceed electively, when the peel may be more mature and comorbidities are optimized. Incidentally, we found that clearing the pleural space with tPA before decortication seemed to make decortication easier and more straightforward.

Besides overall effectiveness, there are several other important findings in these results. First, intrapleural tPA was safe and well-tolerated. There were no instances of hemodynamically significant pleural bleeding or coagulopathic bleeding from other sites to indicate systemic absorption. Therapy was not painful, although some patients with very large effusions experienced pressure during the dwell time (typically after 8-10 hours), prompting early unclamping of the chest tube. Second, treatment was effective despite the use of small-bore catheters. This is consistent with other reports on the use of pigtail catheters for pleural drainage and is important because these catheters can be inserted with image guidance, an easier and safer alternative to insertion of large-bore chest tubes at the bedside. This reduces the risk of procedure-related morbidities, such as pulmonary parenchymal, liver, or spleen injury. Third, effectiveness seemed independent of tPA dose. As reviewed above, MIST-2 and subsequent trials used a higher dose of tPA (10 $\mathrm{mg}$ ). The lower dose used in this study, and elimination of DNase, add up to substantial cost savings. In addition, this suggests that future trials can explore the effectiveness of even $2 \mathrm{mg}$ tPA (the unit dose).

Objective determination of treatment outcome is essential to making valid conclusions about treatment success. Defining treatment failure as referral to surgery, as has been done in many published trials of fibrinolytic therapy, is suboptimal because this is a clinical decision that is subjective and therefore open to bias and individual clinician preference. (Of the seven patients in our study with incomplete drainage, only three went on to surgery.) Another metric has been clearance of opacification on chest x-ray. We used chest CT for outcome assessment in most of our patients because persistent opacification on chest x-ray may be secondary to atelectasis and/or consolidation, not residual fluid. This is common in patients with parapneumonic effusion and should not be considered treatment failure. Surgical therapy does not treat atelectasis and consolidation, and if the effusion is drained, these findings resolve spontaneously with time (Figure 5). A second benefit of chest CT is that if residual loculated fluid is identified, a decision can be made to either continue tPA through the existing chest tube, insert a new image-guided tube for further therapy, or proceed with decortication. In this study, additional tubes were used in $18 / 47$ cases (38\%). 


\section{Cureus}

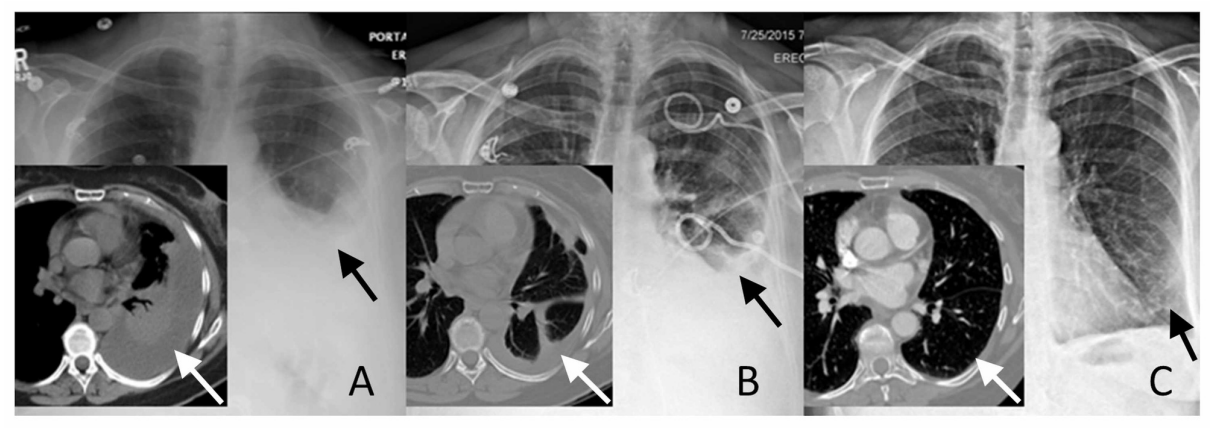

\section{FIGURE 5: Example of Incomplete Drainage Without Surgery}

Representative chest x-rays with CT images inset. A 66-year-old woman presented with several weeks of dyspnea and chest pain. A) Chest $x$-ray suggested a moderate pleural effusion. Chest CT showed left lower lobe atelectasis and consolidation with a parapneumonic effusion. B) A pigtail catheter was placed and she was treated with three doses of tPA. Chest x-ray at completion of therapy showed persistent opacification. Chest CT demonstrated a small residual effusion with a small loculated pocket and with associated pulmonary parenchymal consolidation. The decision was made to conclude therapy and not proceed with surgery. C) Follow-up imaging at one month shows complete resolution of parenchymal and pleural abnormalities. tPA: tissue plasminogen activator.

There are several considerations that limit the ability to draw conclusions from this study. Most important is that it is a retrospective analysis of a group of patients managed on an individual basis and without a clearly defined and implemented protocol. Thus, there is considerable uncontrolled variability in many factors that affect outcome. For example, criteria for determining when to repeat tPA and when to remove chest tubes were not defined in advance. in addition, although chest CT was used to evaluate treatment success or failure in most patients, the application was not uniform and objective measurement of residual effusion volume was not employed. Chest CT should be the gold standard for outcome assessment and simple techniques for quantitation of effusion volume based on CT have been published [19]. The retrospective approach also makes it difficult to determine the impact of tPA therapy on hospital length of stay independent of duration of therapy. Some patients were discharged as soon as the chest tube was removed, while in others chest tubes were left in place while patients were kept hospitalized for other reasons. A prospective protocol defining criteria for repeat treatment, chest tube removal, and determination of treatment failure should streamline therapy, shortening duration of therapy and hospital stays. Another important limitation is that long-term follow-up was not available for many patients, so that it is not possible to know if the short-term successes translated into long-term good outcomes.

\section{Conclusions}

Our study suggests that a short course of low-dose tPA with a longer dwell time and without DNase may be equally, if not more, effective than combination therapy of tPA and DNase with frequent dosing and short dwell times. Our strategy is more feasible and less expensive, but treatment can be prolonged. Based on these findings, we are proceeding with a prospective evaluation.

\section{Appendices}




\section{Cureus}

\section{Microorganism}

Methicillin-resistant Staphylococcus aureus

Methicillin-sensitive Staphylococcus aureus

Fusobacterium nucleatum

Klebsiella pneumoniae

Peptostreptococcus

Eikenella corrodens

\section{TABLE 5: Microorganism Cultured in Pleural Fluid}

Symptom

n

Dyspnea

31

Cough

13

Pleuritic Chest Pain

12

Fever

10

Chest Pain

\section{7}

Flank Pain

Nausea/Vomiting

Fatigue

Obstructed PleurX® Catheter

Loss of Appetite

Abdominal Pain

Back Pain

Hemoptysis

Dizziness

\section{Additional Information \\ Disclosures}

Human subjects: Consent was obtained by all participants in this study. Memorial Healthcare System Institutional Review Board issued approval 002244. This minimal risk study meets the criteria for expedited review as outlined in 45 CFR 46.110(b)(1) or 21 CFR 56.110(b)(1). This study was approved under expedited criteria Category 5. . Animal subjects: All authors have confirmed that this study did not involve animal subjects or tissue. Conflicts of interest: In compliance with the ICMJE uniform disclosure form, all authors declare the following: Payment/services info: All authors have declared that no financial support was received from any organization for the submitted work. Financial relationships: All authors have declared that they have no financial relationships at present or within the previous three years with any 
organizations that might have an interest in the submitted work. Other relationships: All authors have declared that there are no other relationships or activities that could appear to have influenced the submitted work.

\section{References}

1. Light RW: Parapneumonic effusions and empyema. Proc Am Thorac Soc. 2006, 3:75-80. 10.1513/pats.200510-113JH

2. Yu H: Management of pleural effusion, empyema, and lung abscess. Semin Intervent Radiol. 2011, 28:75-86. 10.1055/s-0031-1273942

3. Tillet W, Sherry S, Read C: The use of streptokinase-streptodornase in the treatment of postneumonic empyema. J Thorac Surg. 1951, 21:275-297. 10.1016/S0096-5588(20)31273-3

4. Chin N, Lim T: Controlled trial of intrapleural streptokinase in the treatment of pleural empyema and complicated parapneumonic effusions. Chest. 1997, 111:275-279. 10.1378/chest.111.2.275

5. Davies R, Traill Z, Gleeson F: Randomised controlled trial of intrapleural streptokinase in community acquired pleural infection. Thorax. 1997, 52:416-421. 10.1136/thx.52.5.416

6. Bouros D, Schiza S, Tzanakis N, Chalkiadakis G, Drositis J, Siafakas N: Intrapleural urokinase versus normal saline in the treatment of complicated parapneumonic effusions and empyema. Am J Respir Crit Care Med. 1999, 159:37-42. 10.1164/ajrccm.159.1.9803094

7. Tuncozgur B, Ustunsoy H, Sivrikoz MC, Dikensoy O, Topal M, Sanli M, Elbeyli L: Intrapleural urokinase in the management of parapneumonic empyema: a randomised controlled trial. Int J Clin Pract. 2001, 55:658660.

8. Diacon A, Theron J, Schuurmans M, Van De Wal B, Bolliger C: Intrapleural streptokinase for empyema and complicated parapneumonic effusions. Am J Respir Crit Care Med. 2004, 170:49-53. 10.1164/rccm.200312$17400 \mathrm{C}$

9. Rahman N, Maskell N, West A, et al.: Intrapleural use of tissue plasminogen activator and DNase in pleural infection. N Engl J Med. 2011, 365:518-526. 10.1056/NEJMoa1012740

10. Piccolo F, Pitman N, Bhatnagar R, et al.: Intrapleural tissue plasminogen activator and deoxyribonuclease for pleural infection. An effective and safe alternative to surgery. Ann Am Thorac Soc. 2014, 11:1419-1425. 10.1513/AnnalsATS.201407-3290C

11. Mehta H, Biswas A, Penley A, Cope J, Barnes M, Jantz M: Management of intrapleural sepsis with once daily use of tissue plasminogen activator and deoxyribonuclease. Respiration. 2016, 91:101-106. 10.1159/000443334

12. Maskell N, Davies C, Nunn A, et al.: U.K. controlled trial of intrapleural streptokinase for pleural infection. N Engl J Med. 2005, 352:865-874. 10.1056/NEJMoa042473

13. Tokuda Y, Matsushima D, Stein G, Miyagi S: Intrapleural fibrinolytic agents for empyema and complicated parapneumonic effusions: a meta-analysis. Chest. 2006, 129:783-790. 10.1378/chest.129.3.783

14. Heffner J: Multicenter trials of treatment for empyema - after all these years . N Engl J Med. 2005, 352:926928. 10.1056/NEJMe048352

15. Walker C, Shirk M, Tschampel M, Visconti J: Intrapleural alteplase in a patient with complicated pleural effusion. Ann Pharmacother. 2003, 37:376-379. 10.1345/aph.1C248

16. Skeete D, Rutherford E, Schlidt S, Abrams J, Parker L, Rich P: Intrapleural tissue plasminogen activator for complicated pleural effusions. J Trauma. 2004, 57:1178-1183. 10.1097/01.ta.0000141879.67441.52

17. Froudarakis M, Kouliatsis G, Steiropoulos P, et al.: Recombinant tissue plasminogen activator in the treatment of pleural infections in adults. Respir Med. 2008, 102:1694-1700. 10.1016/j.rmed.2008.08.012

18. Zuckerman D, Reed M, Howington J, Moulton J: Efficacy of intrapleural tissue-type plasminogen activator in the treatment of loculated parapneumonic effusions. J Vasc Interv Radiol. 2009, 20:1066-1069. 10.1016/j.jvir.2009.04.067

19. Moy MP, Levsky JM, Berko NS, Godelman A, Jain VR, Haramati LB: A new, simple method for estimating pleural effusion size on CT scans. Chest. 2013, 143:1054-1059. 10.1378/chest.12-1292 